\title{
Die Lösungsverhältnisse bei Pneumonia fibrinosa und Pneumonia tuberculosa sive caseosa.
}

\author{
Von \\ Dr. V. Schläpfer, \\ Assistent am pathol. Institut.
}

Zu denjenigen Krankheitsbildern, die durch die quantitativen Umsetzungen, welche sich in ihrem Verlaufe vollziehen, vor allem die Aufmerksamkeit des Klinikers, Pathologen und Physiologen auf sich lenken, gehört unter andern zuerst das der Pneumonia fibrinosa. Die in wenigen Tagen in der Lysis sich vollziehende Gewichtsverminderung vom Stadium der grauen Hepatisation zu dem der vollzogenen Resolution, ist eine der auffälligsten, quantitativen, chemischen Leistungen, die die Biologie des menschlichen Organismus aufzuweisen hat. - Durch diese Lösung des Exsudates steht die Pneumonia crouposa im schroffsten Gegensatz zu einem in gewissen Beziehungen ähnliche Symptome ${ }^{1}$ ) aufweisenden, ebenfalls entzündlichen Lungenprozess, der Pneumonia tuberculosa sive caseosa, die geradezu durch das Fehlen dieser Erscheinung pathologisch-anatomisch gekennzeichnet ist. Denn das in vielen Fällen auch beträchtliche Exsudat bleibt hier bestehen und verwandelt sich aus bisher noch nicht einwandsfrei festgestellten Gründen in die eigentümliche, an Käse erinnernde Masse um, deren Vorhandensein im allgemeinen mit ziemlicher Sicherheit einen tuberkulösen Prozess vermuten lässt.

Die Ursache dieses verschiedenartigen Verhaltens scheint auf den ersten Blick auf der Hand zu liegen. Es ist zweifellos die Verschiedenheit der Krankheitserreger, des Diplococcus pneumoniae und des Bacillus tuberculosis, $\mathrm{zwei}$ in ihren Lebensäusserungen relativ sehr voneinander abweichenden Mikroorganismen. Allein trotz der

1) v. Mering, Lehrbuch der inneren Medizin. II. Aufl. 
Annahme, die Lösungsverhältnisse seien durch die Spezifität der Krankheitserreger bedingt, ist damit im Grunde genommen doch keine eigentliche Erklärung gegeben. Denn erstens kann wohl die Spezifität das Grundmoment sein, allein der Weg, anf dem sich deren Einfluss zeigt, hietet verschiedene Richtungen, zweitens kann trotz des Vorhandenseins der beiden Bakterien in beiden Fällen doch einmal die Lösung des pneumofibrinösen Exsudates ausbleiben oder die Resolution der pneumotuberkulösen Entzündungsprodukte eintreten, d. h. die Reaktion des Organismus auf die Bakterienwirkung kann variieren, und drittens ist Verkäsung wohl ein sehr wahrscheinliches, allein nicht monopolisiertes Kriterium der Tuberkulose, indem sie auch bei nicht tuberkulösen Krankheitsprozessen, wie der Lues z. B., auftritt.

Es ist deshalb von verschiedenen Seiten der Wunsch geäussert und der Versuch gemacht worden, nach einem weitern oder vielmehr tiefern Kausalmoment für die Lysis zu suchen. Denn während man sich in der rein bakteriologischen Ära mit dem Hinweis auf die Verschiedenartigkeit der Bakterien zufrieden geben konnte, wurde durch die in neuerer Zeit wieder mehr in den Vordergrund getretenen Forschungen, die mehr die dem Organismus eigenen Schutzkräfte berïchsichtigen, der Wortlaut der Frage etwas anders gestellt. Die Untersuchungsergebnisse über autolytische Prozesse berechtigen dazu, die Lösung der Aufgabe auch auf diesem Wege zu versuchen.

Von Aufrecht, Naunyn, Hofmeister u. a. wurde zum ersten Male deutlich ausgesprochen, dass man unter dem Resolutionsvorgang der Pneumonia fibrinosa einen bestimmten peptonisierenden Chemismus verstehen müsse. In seiner Arbeit „Über die Lösungsvorgänge bei der krupösen Pneumonie" modifiziert Simon") diesen peptonisierenden Prozess dahin, dass er ihn unter den von Salkowsky, Hofmeister, Jacoby etc. näher präzisierten Begriff der Autolyse stellt und S. 623 als höchst wahrscheinlich annimmt, dass „wenn irgendwo im Körper absterbendes Gewebe sich findet, in den absterbenden Zellen Vorrichtnngen ausgebildet werden, die einen vollständigen Zerfall des abgetöteten Materials einleiten". Solch absterbendes Gewebe aber wäre nach $\mathrm{ihm}$ das pneumonische Exsudat. Da nun dieses aber nicht nur aus Zellen, sondern auch aus nicht organisiertem Fibrin besteht, so ist die Si monsche Erklärung streng genommen nicht ganz befriedigend. Die fehlende Lücke nun wird vollkommen ergänzt durch die Anschanung Friedrich Müllers ${ }^{2}$ ).

1) Simon, Untersuchungen über die Lösungsvorgänge bei der krupösen Pneumonie. Deutsch. Archiv f. klin. Medizin. LXX. Bd. p. 604-623. 1901.

2) Friedrich Müller, Über die Bedeutung der Selbstverdaung bei einigen krankhaften Zuständen. Verhandl. d. Kongr. f.-innere Med. Wiesbaden 1902. 
Dieser Autor hatte schon 1884 den Nachweis erbracht, dass den Eiterkörperchen verdauende Eigenschaften zukommen auf Grund eines peptonisierenden Fermentes, das bei ihrem Zerfall frei werden soll. Da nun im Exsudate der Pneumonie Leukozyten zu Beginn der Resolution das Hauptkontingent der vorhandenen Exsudatmasse bilden, so lässt es Fr. $M$ ü ll e r als wahrscheinlich erscheinen, dass die Lösung vor allem auch des Fibrins eben auf Grund dieser verdauenden Eigenschaften eine Arbeit der Leukozyten sei. Das Moment aber, das den Zerfall der Leukozyten begünstigt, und das somit primärer Natur sein muss, lässt auch er, wie Simon, unberücksichtigt. Wahrscheinlich identifizierten sie dasselbe mit der durch die krankheitserregenden Mikroorganismen verursachten Schädigung.

Beide Autoren aber glauben in ihren den modernsten Standpunkt vertretenden Arbeiten auch sicher annehmen zu dürfen, dass die lösenden Eigenschaften in keiner Beziehung dem Diplokoccus zugeschrieben werden können.

Nicht so abgeklärt sind die Verbältnisse bei der Pneumonia tuberculosa sive caseosa. Dass der Tuberkelbazillus keinen peptonisierenden Einfluss ausübt, wird bei den hier vorliegenden Umständen nicht weiter in Frage gestellt werden müssen. Die Aufgabe lautet vielmehr so, $\mathrm{zu}$ untersuchen, warum hier eine Lösung ausbleibt; es ist dies etwas überraschend, da es sich beim pneumotuberkulösen Exsudate ebenfalls um absterbendes Gewebe handelt, das nach Si mon ohne weiteres dem Zerfall verfallen sein müsste. Man könnte sich fragen, 1. ob der Tuberkelbazillus imstande ist, die als Käse imponierende typische Gewebsveränderung als spezifische Funktion hervorzubringen, oder 2. ob gewisse, durch die Wirkung des Tuberkelbazillus hervorgerufene Schädigungen bestimmter Gewebselemente, wie z. B. der Blutgefässe, gleichsam als sekundäre Momente dem Phänomen zugrunde liegen, oder 3 . ob schliesslich das tuberkulöse pneumonische Exsudat eine wesentlich andere zelluläre Zusammensetzung besitzt, vielleicht eine Armut an Leukozyten, die ja nach Müller bei der Lysis im Vordergrund stehen.

Auf dem Boden der sub 1 genannten Anschaung steht $V$ is smann ${ }^{1}$, der die Verkäsung als spezifisches Produkt der durch die Tuberkelbazillen erzeugten Stoffwechseltoxine hinstellt. Ihm nahe kommt Baumgarte ${ }^{2}$ ), nach welchem das Alveolarexsudat (S. 380)

1) Vissmann, Wirkung toter Tuberkelbazillen und des Tuberkulins auf den tierischen Organismus. Virch ow s Arch. 129. Bd. 1892.

2) Ba u m g r ten, Bemerkungen zur Lehre von der käsigen Pneumonie mit besonderer Berücksichtigung von Orths Abhandlung über dieselbe. Beitr. zur path. Anat. 1. Bd. $1891,92$. 
unter dem Einfluss der Bazillen verkäst, während $\left.\mathrm{Orth}{ }^{1}\right)$ entsprechend der sub 2 erwähnten Möglichkeit (S. 54) die „Undurchlässigkeit der Kapillaren" als eine der Ursachen für die Verkäsung ansieht, wobei aber doch "die Hauptsache in den Bazillen zu sehen sei“. Über die sub 3 genannte Eventualität sind die Forschungsergebnisse noch am wenigsten ausgeprägt. Während nach Wolf (S. 7) die Tuberkeltoxine speziell gegenüber Leukozyten chemotaktisch negativ sein sollen, hält B a umgarten ${ }^{2}$ z. B. das Tuberkulin Koch (S. 26), für eine in dem genannten Sinne chemotaktisch positive Substanz. Sowohl nach Orth aber als auch nach $B$ a u m garten, M ets chnik of $\left.f^{3}\right)$, Herxhei mer ${ }^{4}$ ) u. A. finden sich in tuberkulös-pneumonischen als auch in den übrigen tuberkulösen Infiltrationsherden neben anderen Zellarten auch Leukocyten, die eben wie das übrige auch der Verkäsung anheimfallen. Ihre peptonisierende Wirkung kann nach Orth und Baumgarten erst zur Wirkung kommen, wenn die Virulenz des tuberkulösen Virus erloschen ist, d. h. bei der Mazeration und zwar nicht als Funktion der schon vorhandenen, sondern von solchen Leukozyten, die in Einwanderung begriffen sind. DieFrage nach der zellulären Beteiligung am tuberkulösen Infiltrationsprozess muss daher noch offen bleiben. Doch scheint mir dies für die in dieser Untersuchung verfolgten Zwecke weiterhin nicht ron Belang zu sein, weil ja auch bei der krupösen Pneumonie die Anwesenheit der Leukocyten kombiniert mit der Si mon schen Hypothese immer noch keine vollkommen befriedigende Antwort gibt, wie oben angedeutet wurde, indem die tiefliegende Ursache des Verfalls dadurch nicht gegeben wird. Vor allem aber ist dadurch nicht erklärt, warum nicht in allen Fällen von Pneumonia crouposa eine Lösung eintritt, da doch stets grosse Mengen von Leukozyten zur Verfügung stehen. Deshalb wird auch für die Pneumonia tuberculosa diesem Faktor nicht eine allzu dominierende Bedeutung zugeschrieben werden dürfen.

Man darf daher wohl behaupten, dass weder die Frage über das Verhalten des Exsudates bei Pneumonia fibrinosa noch die Momente bei der Entstehung des tuberkulös käsigen Entzündungsproduktes vollständig befriedigend sichergestellt und gelöst sind.

1) Orth, Welche morphologischen Veränderungen können durch Tuberkelbazillen erzeugt werden? Verhandl. d. deutsch. path. Ges. Hamburg. 1901.

2) B a umgarten, Über die pathologisch-histologische Wirkung und Wirksamkeit des Tuberkelbazillus. Verhandl. d. deutsch. pathol. Gesellschaft. Hamhurg 1901.

3) Weigert, Über Metschnik offs Theorie der tuberkulösen Riesenzellen. Fortschritte der Medizin. 6. Bd. 1888. Nr. 21.

4) Herxheimer, Über die Wirkungsweise des Tuberkelbazillus bei ex. perimenteller Lungentuberkuluse. Zieglers Beitr. 33. Bd. 1903. 
Deshalb nun und namentlich, weil noch viele Widersprüche als unerledigt $z u$ betrachten sind, schien mir ein weiterer Versuch, die Aufgabe zu lösen, eine gewisse Berechtigung zu haben, um so mehr, da der Standpunkt, von dem aus das Problem in Angriff genommen werden soll, ein von dem der genannten Autoren etwas abweichender ist und namentlich den nach der früheren Auseinandersetzung zutage tretenden Gegensatz zwischen Pneumonia fibrinosa und tuberculosa zu ïberbrücken imstande ist. Die Gegenüberstellung der beiden Krankheitsprozesse geschah daher mit Absicht. Denn meines Erachtens gibt gerade das gegensätzliche Verhalten dieser zwei Krankheitsformen gewissermassen einen Anhaltspunkt zur Erklärung, indem sie gleichsam die experimentellen Extreme darstellen, die bei Berücksichtigung des näher zu beschreibenden Momentes in Frage kommen könnten.

Damit aber ist angedeutet, dass das Prinzip, das dem folgenden Erklärungsversuch zugrunde liegen soll, tiefer liegt und auf allgemeinerer Basis als das in den zitierten Arbeiten zutage tretende, indem die Fragestellung nunmehr lautet: Was führt einen Zellabbau überhaupt herbei?

Bei Untersuchungen über das Verhalten des Sauerstoffes in der lebenden Zelle und bei ihrem Absterben ${ }^{1}$ ) ergab sich das eigenartige Resultat, dass das Leben der Metazoenzelle sich bewegt zwischen der tödlichen Autolyse infolge grosser Sauerstoffzufuhr und der Zersetzung als Folge der Erstickung oder des Sauerstoffmangels. Während bei Erstickung die Gewebe ein eigentümlich opakes Aussehen annehmen und in eine kompakte fettähnliche Masse übergehen, die sich bei sehr langer Aufbewahrung weiterhin nicht mehr verändert, und erst wenn Sauerstoff zutritt, in körnigen Zerfall gerät, findet bei überreichem Sauerstoffzutritt eine sehr rasche, oxydative Zersetzung statt, die bei nicht genügendem Stoffersatz in kürzester Zeit den Tod herbeiführt und ungeschwächt weiter schreitet. Die Gewebe zerfallen in körnigen Detritus, der teilweise in Lösung zu gehen scheint, so dass seine Formelemente dem mikroskopischen Sehen nicht mehr weiterhin zugänglich bleiben. Das so erhaltene Bild gleicht in gewisser Beziehung dem, das man durch künstliche, unter Sauerstoffabschluss vollzogener Trypsinverdauung erhält. Das heisst mit andern Worten: Sauerst offarmut oder Mangel wirkt an und für sich für die Gewebe konservierend, indem dieselben wohl ihr strukturelles Bild in gewissem Grade verlieren; aber die Volumverhältnisse bleiben bei verhin-

1) Schläpfer, Ửber den Bau und die Funktion der Epithelzellen des Plexus chorioidens. in Beziehung zur Granulalehre und mit besonderer Berücksichtigung der vitalen Färbungsmethoden. Z i eglers Beitr. VII. Suppl. 1905. 
derter Eintrocknung mehr weniger konstant und die äusseren Grenzen sind gewahrt. Sauerstoffanwesenheit oder Reichtum dagegen führt eine körnige Auflösung der Zelle und der Gewebe herbei, eine Tatsache, die auch während des Lebens eintritt, durch anderevitale Prozesse aber fortwährend kompensiertwird, bevorein tödlicher Grad erreicht ist. Wenn daher jene Prozesse geschädigt sind, ist die Wirkung des Sauerstoffs die allein massgebende. Man darf deshalb sagen, dass absterbende und erstickte Gewebe auch ohne Mithilfe typischer peptonisierender Fermente durch Sauerstoff allein bis zu einem gewissen Grade abgebaut werden können und dass dies auch, falls keine besonderen Vorkehrungen getroffen sind, in der Regel eintritt.

Überträgt man nun diese Sätze auf die hier zu besprechenden zwei Krankheitsbilder, so müsste man den Schluss ziehen: Bei Pneumonia fibrinosa ist Sauerstoff vorhanden, bei Pneumonia tuberculosa fehlt er, falls man nicht die Möglichkeit von im einen Fall vorhandenen, im anderen fehlenden peptonisierenden Fermenten einräumen will. Für eine solche Annahme aber, die ja an und für sich nur eine veränderte Fragestellung bedeuten würde, lässt sich kein zwingender Beweis finden. Denn die in diesem Sinne vorgenommenen Arbeiten von Fr. Müller und Simon fielen negativ aus, indem sie nur zugunsten eines intrazellulären autolytischen oder nach dem Zerfall freiwerdenden, also erst sekundären Katalysators sprechen, dessen Bedeutung für diese Frage oben bereits erörtert worden ist. Wollte man aber doch das Vorhandensein eines primären lösenden Fermentes in Erwägung ziehen, so müsste dasselbe, da einmal das Peptonisierungsvermögen der Leukocyten nicht befriedigend aufklärt und die pathogenen Mikroben völlig ausser Betracht fallen, vom Lungenparenchym sezerniert werden. Alsdann müsste aber gerade bei der tuberkulösen Pneumonie Lösung eintreten, da vor allem ein intensiv geschädigtes Gewebe, wie es speziell bei der Tuberkulose entsteht, durch solche Fermente verdaut werden muisste. Gegen den Einwand aber, dass eben bei der Pneumonia tuberkulosa diese Fermente unwirksam seien, weil sie die Tuberkeltoxine zu lähmen vermögen, wäre anzaführen, dass doch auch bei virulenter Tuberkulose gelegentlich Lösung eintritt, ausserdem aber nichts für eine solche fermentsezernierende Fähigkeit des Lungengewebes zu sprechen vermag und Verkäsung ausserdem (s. o.) kein Spezifikum der Tuberkulose ist, sondern auch bei anderen Krankheiten vorkommt, die vor allem mit einer Zerstörung der Gefässe verbunden sind, ein Faktor, der namentlich gegen die Auffassung spricht, käsige Massen seien ein Produkt der spezifischen Tätigkeit der Tuberkelbazilen (s. o.). 
In den bisherigen Erklärungsversuchen hatte Fr. M ii ller hauptsächlich auf die Bedeutung der Leukozyten aufmerksam gemacht, Simon ging um einen Schritt weiter und glaubte durch Zuziehung des autolytischen Vermögens der Gewebe das Fehlende zu ergänzen. Allein auch damit ist, wie angeführt, die Lösungsfrage nicht erschöpfend beantwortet; die Erklärung stösst namentlich auf Schwierigkeiten bei den im Verlauf etwas atypischen Pneumonien, wie z. B. der Karnefikation, zudem aber kann die Autolysetheorie von Sim on a priori nicht genügen, weil nach Schlesinger ${ }^{1}$ ) unter Autolyse wohl ein bestimmter charakteristischer abbauender Prozess verstanden werden muss, die Ursache aber, die eine Verlangsamung oder ein Sistieren desselben herbeiführt, was speziell hier von Wichtigkeit sein muss, nicht bekannt ist.

Man wird daher wiederum, wenn man ins Auge fasst, dass 1. bei derselben Krankheit das eine Mal Lösung eintritt, das andere Mal ausbleibt und dass 2. Verkäsung durch verschiedene Ursachen in den meisten Organen hervorgerufen werden kann, zu der Vermutung geführt, dass, wie schon Sim on betont, diesen Phänomenen ein allgemeines Prinzip zugrunde liegen muss, und zwar meiner Ansicht nach sehr wahrscheinlich jenes, welches bei der Autolyse eine Verlangsamung oder ein vollständiges Erlöschen verursachen kann, da es sich ja hier im Grund genommen um nichts anderes handelt als um Eintreten oder Ausbleiben der Autolyse. Ein solches allgemeines Prinzip aber wäre nach obigem der Sauerstoff.

Dieser wird den Geweben durch das Blut zugeführt, das ihn speziell in den Kapillaren abgibt.

Wenn deshalb die Behauptung betreffs die primäre Bedeutung des Sauerstoffs für das Verbalten der Pneumonia fibrinosa und tuberculosa richtig sein soll, wird die nächste Konsequenz, wie schon angedeutet, die sein, dass bei der einen 0 zugeführt werden muss, bei der andern fehlt, $d$. h. dass die Kapillarzirkulation bei der einen vorhanden, bei der andern aufgehoben ist. Untersucht man daraufhin die beiden Gewebe in Schnitten, so erhält man die pathologischhistologisch bekannten Bilder, die sich bei näherer Betrachtung wie gewünscht in das Schema einfügen lassen.

Bei der Elastinfärbung nach Weigert ergibt sich für die Pneumonia caseosa bekanntlich ein vollkommener Verlust jeder strukturellen Anordnung, die elastischen Fasern des Lungenparenchyms sowohl als

1) Schlesinger, Untersuchungen über die Abhängigkeit der autolytischen Prozesse von physiologischen Verhältnissen. Hofmeister Beiträge $z$. chem. Physiologie. 1904. 87-115. 
die Gefässe gehen wohl bis an die scharf markierte Grenze der Infiltration heran, im Bezirk selbst aber lassen sich nur spärliche Reste entdecken. Umgekehrt weist auch die dicht infiltrierte kruppös-pneumonische Lunge stets deutlich den alveolären Aufbau auf, wobei auch die Kapillaren stets scharf in die Erscheinung treten, namentlich aber bei Hämatoxylin-Eosinfärbung nach Delafield, wo sie vermöge ihres Gehaltes an Erythrozyten sich besonders kenntlich machen, während die tuberkulöse Infiltration anch bei dieser Methode sich völlig negativ verhält.

Während also demnach bei Pneumonia caseosa jede Durchblutung völlig ausgeschlossen ist, ist die Zirkulationsmöglichkeit bei Pneumonia fibrinosa auf Grund des histologischen Bildes ohne weiteres einzuräumen. Der direkte Beweis, dass in dem derb infiltrierten Gewebe in der Tat eine Blutdurchströmung stattfinde, ist allerdings kaum zu erbringen, allein eine gewisse biologische Überlegung scheint mir hier in gewissem Sinne das Fehlende befriedigend ergänzen zu können. Es wäre nämlich schwer verständlich, wie, falls eine vollständige Stase während der Zeit der roten und grauen Hepatisation vorhanden wäre, nach Ablauf der immerhin im Minimum 3-4 Tage dauernden Störung sich eine vollständige Restitutio ad integrum ausbilden. könnte. Denn in den meisten Fällen müsste doch eine Stauung der Zirkulation schon in kürzerer Zeit zum Absterben der betroffenen Gewebe führen und das um so mehr bei dem sowieso durch die Infektion geschädigten Lungenparenchym. Diese Überlegung samt dem histologischen Befund, glaube ich, mag die Berechtigung zur Annahme einer wenn auch etwas herabgesetzten Zirkulation in den pneumonisch entzündeten Lungenpartien zur Genüge decken. Es ergibt sich also somit die Schlussfolgerung:

Bei Pneumonia fibrinosa sind die Gefässe relativ wenig geschädigt, d. h. der Diplococcus pneumoniae zeigt in dieser Beziehung keine grosse Pathogenität; sie erlauben dabei eine, wenn auch etwas gestörte Zirkulation. Die Sauerstoffzufuhr ist deshalb vorhanden. Da aber die in unverhältnismässig grosser Zahl vorhandenen intraalveolären Zellen, Epithelien und Leukozyten, einmal durch die Infektionserreger geschädigt, sodann aber auch durch die in jeder Beziehung ungenügende Ernährung im Absterben begriffen sind, tritt in $\mathrm{Be}-$ ziehung zu den den Zerfall kompensierenden intrazellulären Prozessen ein Sauerstoffüberschuss, d. h. eine zu rasche oxydative Autolyse ein, die in kurzer Zeit den körnigen Zerfall herbeiführen muss. Rote Blutkörperchen, Epithelzellen und Leukozyten lösen sich nun in körnigen Detritus auf, wobei aus weissen Blutzellen polymorphkerniger Art 
die peptonisierenden Fermente frei werden, die nun auch das noch restierende Fibrin zur Lösung führen.

Soll nun desgleichen das histopathologische Verhalten der Pneumonia tuberculosa auf das Verhalten des Sauerstoffs zurückgefübrt werden, so muss also vor allem die Zirkulation ausgeschlossen werden. Und zwar mass die Zirkulationsstörung primärer Art sein, d. h. es müssen unter allen Gewebsstrukturelementen zuerst die Kapillaren durch den Tuberkelbazillus vernichtet werden. Denn vorausgesetzt, die Kapillarnekrose sei sekundärer Natur, so müsste man darunter ein Ergriffenwerden der Kapillarendothelien etc. durch die die Verkäsung erzeugenden Tuberkeltoxine verstehen. Damit wäre aber eingeräumt, dass die Käsemassen durch spezifische Tätigkeit der Tuberkelbazillen entstehen, z. B. also, wie oben pag. 46 bemerkt, durch Abtöten der zur Autolyse führenden Fermente. Die Rolle des Sauerstoffmangels aber würde ausser Betracht fallen. Mit der Frage aber, ob der Tuberkelbazillus in der Tat zuerst bei seinem Eintritt in die Gewebe die Kapillaren schädigt, wird ein Gegenstand berührt, der eben noch im Zentrum der eifrigsten Diskussion gestanden hat. Während B a u m g arten (s. o.) vor allem betont, dass der eingedrungene Tuberkelbazillus zuerst Proliferation der fixen Bindegewebszellen und erst sekundär Absterben der Kapillarendothelien erzeuge, treten Wech sberg (s. o.), Herxheimer (s. o.), Miller (s. o.) und andere für eine primäre Schädigung der Kapillaren ein und Weigert (s. o.), Orth (s. of, Rosthenisch (s. o.), Volkow (s. o.) und Martin (s. o.) definieren Verkäsung als Nekrose infolge Mangel an Gefässen, allerdings ohne eine nähere, tiefergehende Erklärung anzuführen. Eine endgültige Entscheidung in diesem Falle kann selbstverständlich nur das Experiment bringen, wie es in ziemlich unanfechtbarer Weise von Herxheimer (s. o.) ausgeführt worden ist. Allerdings hat er durch eine intravenöse Injektion teilweise seiner Anschauung entgegengearbeitet, ein Vorwurf, den Baumgarten namentlich gegen die Arbeit W e chsbergs (s. o.) erhebt, indem er darauf aufmerksam macht, dass bei der Injektion schon eine mechanische Läsion durch die fortgeschwemmten Partikel berücksichtigt werden müsse. Allein gerade hier ist der Grund zu diesem Vorwurf besonders sorgfältig berücksichtigt worden, indem nur ganz feinste Kultursuspensionen verwendet wurden. Es ist deshalb nicht anzunehmen, dass der ins Blut strömende Tuberkelbazillus die Kapillarendothelien vor allem zu schädigen vermöge, weil er mechanische Läsion verursache, sondern vielmehr auf Grund einer spezifischen Giftwirkung. Dann aber ist begreiflich, dass er dies auch tun kann, wenn er z. B. auf dem Lymphwege oder durch das Bronchiallumen transportiert wird. 
Infolge dieser ziemlich einwandsfreien Arbeit Herxheimers glaubte ich daher von eigenen Experimenten in diesem Sinne absehen zu dürfen, um so mehr, weil einige durch zufällige Untersuchungen tuberkulöser Organe gewonnenen Bilder sehr zugunsten der $\mathrm{Herx}$ heimerschen Arbeit sprechen.

Es handelte sich im einen Fall um eine akute Tuberkulose des Peritoneums, speziell des Omentum majus, das andere war ein Präparat einer miliaren Lungentuberkulose mit Gefässinjektion, das mir Herr Professor Dr. Ernst in dankenswertester Weise zur Verfügung stellte. Bei beiden war vor allem die Tatsache charakteristisch, dass die Gefässe um den Sitz des tuberkulösen Virus in einer Zone um die Riesenzelle wie abgeschnitten erschienen.

Im zweiten Fall war die Verkäsung schon in hohem Grade ausgebildet, und es liess sich nicht entscheiden, ob die Gefässnekrose primärer Natur war oder Hand in Hand ging mit der allgemeinen Nekrose. Das erste Präparat dagegen war bei weitem klarer. Es war wohl ebenfalls eine ringartige, gefässfreie Zone scharf gezeichnet bemerkbar um die Riesenzelle, allein das Parenchymgewebe war noch deutlich zu unterscheiden, und wenn man die Kernfärbung als Zeichen einer gewissen Intaktheit ante mortem gelten lassen will, mit noch relativ wenig geschädigten Zellen. Es ist dies in Anbetracht der grossen Gefässzerstörung etwas überraschend und könnte deren Bedeutung als ätiologisches Moment der Verkäsung beinahe in Frage stellen; doch lässt sich dieser Widerspruch leicht lösen, wenn man bedenkt, dass die vorliegende Form der Miliartuberkulose akutester Art war, so dass die Zeit vom Eintritt der Kapillarschädigung bis zum Tod zur Ausbildung einer typischen Verkäsung nicht genügen konte.

Auf Grund der angeführten Tatsachen scheint es mir daher erlaubt anzunehmen, dass der Tuberkelbazillus vor allem die Kapillaren zu schädigen vermöge. Die Folge dieser primären Kapillarschädigung ist neben mangelnder Ernährung auch Aufhören der Sauerstoffzufuhr und die weitere Konsequenz hiervon, um mit Weigert, Oṛth, Rostenisch, Volkow u. a. (s. S. 49) zu reden, Nekrose und zwar speziell Verkäsung, wie sie neben Tuberkulose auch noch bei anderen krankhaften Prozessen (s. o.) sich zeigt. Während bei der Pneumonia fibrinosa nur die Ernährung gestört war, Sauerstoff aber noch vorhanden, fehlt nun hier bei der Pneumonia tuberculosa, deren Verhalten für alle tuberkulösen überhaupt mit Verkäsung einhergehenden Prozesse gelten dürfte, beides. Eine Überladung mit Sauerstoff oder vielmehr eine im Verhältnis zur Grösse der restituierenden Prozesse allzu grosse Intensität der abbauenden Oxydation kann daher hier nicht eintreten, d. h. das durch die Tuberkulosetoxine geschädigte 
Gewebe, die toten Leukozyten, histioiden Zellen, fixen Bindegewebszellen, die abgestorbenen Alveolarepithelien etc. können keinen weitern Zerfall eingehen. Bei der im Körperinnern herrschenden Temperatur verwandeln sie sich vielmebr bei Abwesenheit anderweitiger Störungen, wie z. B. Fäulnisbakterien, in jene eigentümliche Masse, die Käse genannt wird und die in sehr ähnlicher Art beim Aufbewahren aseptischer Organe unter Sauerstoffabschluss gewonnen wird, durch eine Art Autolyse bei Sauerstoffmangel. Man kann hiergegen einwenden, dass diese Erklärung nicht genügen könne, weil im Verbältnis zu den sehr häufig sich vorfindenden abgestorbenen Gewebspartien Verkäsung relativ selten sei. Allein dieser Einwurf lässt sich widerlegen. Wenn z. B. ein Thrombus oder besser ein Infarkt entsteht, so handelt es sich in der Tat auch um abgestorbenes Gewebe. Allein die Nachbarschaft dieses toten Bezirkes weist sehr rasch Proliferation auf. Es dringen Kapillarsprossen in die Thrombus- resp. Infarktmasse hinein. Die Blut- und damit die Sauerstoffzufubr beginnt. Das zunächst liegende Gewebe zerfällt und so schreitet der Abbau weiter bis zur vollständigen Resorption. Anders dagegen verhält sichs bei der Tuberkulose. Hier sind im toten Gewebe, das durch eigenartige Umsetzungen auch autolytischer Natur jeden strukturellen Charakter völlig eingebüsst hat, die schädigenden Toxine vorhanden, jedem Eindringen von Kapillarsprossen in den Herd ist daher ein Riegel gesetzt und eine Resorption bleibt aus. Erst wenn Sauerstoff dazutritt, wie bei der Lunge z. B. dann, wenn die Verkäsung einen grösseren durchgängigen Bronchus erreicht, oder wenn die Toxizität des Herdes abgeschwächt ist und Gefässwucherung stattfinden kann, tritt der weitere Zerfall ein. Man könnte hier entgegnen, dieser zersetzende Einfluss des Sauerstoffs bei der Verkäsung könne nicht bedeutend sein, da ja nach Schmoll und Sonn (s. Müller [s. o.] pag. 194) im Brutofen aufbewahrte Käsemassen trotz der Anwesenheit von Sauerstoff nicht wesentlich zerfallen. Allein man muss bedenken, dass der Käse eben eine kompakte Masse ist, der Sauerstoff daher nur oberflächlich Zutritt hat, und da das zerfallende Material nicht wie im Körper wegtransportiert wird, sich auf die Oberfläche beschränken muss. Dies Moment des fehlenden Sauerstoffs bietet vielleicht auch eine Erklärung für die von Schlesinger (s. o.) beobachtete, noch aus unbekannter Ursache eintretende Verlangsamung und Sistierung der autolytischen Prozesse aseptisch aufbewahrter Organe, indem. diese, obschon noch ein grosser Teil unverändert ist, plötzlich abschwächen und erlöschen. Es ist dies verständlich, wenn man bedenkt, dass wegen der Sterilisation die Autolyse in der Regel in dicht verschlossenen Gefässen, zum Teil auch in Flüssigkeiten, vorgenommen wird, also mit 
begrenzter O-Menge. Umgekehrt erklärt Sauerstoffanwesenheit wiederum die nach Orth (s. o.) mangelnde Verkäsung bei tuberkulösen Prozessen geringer Virulenz, da die Kapillaren dort wenig geschädigt sind. Was aber das wichtigste zu sein scheint, ist, dass unter Berücksichtigung der Rolle des Sauerstoffs ohne weiteres verständlich wird, warum auch bei gewissen nicht tuberkulösen pathologischen Prozessen Verkäsung auftritt. Es handelt sich eben auch wie bei der Lues z. B. um Vorgänge, deren Erreger eine spezifische Noxe für die Kapillargefässe bedeuten.

Manche Bilder sprechen gelegentlich beinahe dafür, dass der Organismus das Bestreben hat, in das erkrankte tuberkulöse Gewebe Gefässe zu treiben und dass dieselben aber gleichsam wie durch eine Noxe automatisch gelähmt und geschädigt werden.

Mit der Annahme des lösenden Einflusses der Sauerstoffanwesenheit und den Konsequenzen des Sauerstoffmangels lässt sich meiner Ansicht nach eine ganz erschöpfende, einwandsfreie Erklärung der verschiedenen anatomisch-klinischen Bilder der Pneumonia caseosa und fibrinosa geben. Wenn man auch berücksichtigt, dass die zelluläre Zusammensetzung der beiden Entzündungsprodukte wesentlich voneinander abweicht, so spricht dies nicht gegen die primäre Bedeutung des Sauerstoffes, wie schon oben auseinandergesetzt worden ist. Es scheint mir daher auch nicht von besonderem Belang zu sein, dass eben die zytologische Analyse, namentlich des tuberkulösen Exsudates, noch sehr unvollkommen ist, um so mehr, da immerhin festgestellt ist, dass die Beteiligung der polymorphkernigen Leukozyten an der $\mathrm{Zn}$ sammensetzung des pneumo-fibrinosen Exsudates bedeutend viel grösser ist als an der des pneumo-tuberkulösen. Wie auch schon erwähnt, trägt dieser Umstand unter Zuhilfenahme der M ülle rschen Theorie namentlich dazu bei, die Lösung des in den meisten Fällen doch reichlicheren Fibrinmaterials bei der ersteren gegenüber der letzteren verständlich zu machen, da die lytische Rolle des Sauerstoffs vorderhand nur für zellulär organisierte Substanzen sichergestellt ist.

Die Zusammenfassung und Beantwortung der gestellten Fragen würde also lauten: Das klinisch-anatomisch verschiedene Verhalten der beiden Pneumonieformen ist bedingt durch ihre Beziehung zum Sauerstoff, d. h. zur Zirkulation in den Kapillaren oder mit anderen Worten, durch die Wirkung ihrer Erreger auf die Gefässe. Die anfangs gemachte Behauptung, der blosse Hinweis auf die Spezifität der Erreger sei keine genügende, überhaupt keine Erklärung, muss also dahin modifiziert werden, dass vor allem die Indifferenz des Diplococcus pneumoniae gegen die Kapillarendothelien die Benignität der durch ihn verursachten Erkrankung, die spezifische Pathogenität des Tuberkelbazillus aber für die Kapillarzellen die Malignität der Tuber- 
kulose erkläre. Indifferenz aber und Affinität sind Produkte der Faktoren: Virulenzgrad des Mikroorganismus, Widerstandskraft des Körpers und immerhin auch durch die Regelmässigkeit des Auftretens wahrscheinlich gemacht eine spezifisch-chemische Verschiedenheit der Toxine. Von diesen drei ist der letzte der wichtigste, noch unbekannter Natur. Daher ist auch im Grunde genommen die Antwort auf die gestellten Fragen ungenügend.

Allein trotz dieser Unzulänglichkeit der Erklärung, die schliesslich auch auf der Unkenntnis jener Faktoren beruht, die die Affinität der Elemente und Verbindungen bedingen, erleichtert die Berücksichtigung der Bedeutung des Sauerstoffes in der Biologie doch das Verständnis der beiden in Frage gestellten Prozesse, sei es in ihrer charakteristischen Art, sei es in den mannigfaltigen, klinisch-pathologischen Variationsformen derselben, und bietet meiner Ansicht nach dadurch auch gewisse Fingerzeige für die Therapie.

Die Pneumonia fibrinosa gibt bekanntlich die beste Prognose dann, wenn sie unter sonst gleichen Umständen junge kräftige Personen ergreift, deren Herz- und anderes Gefässsystem über ein gewisses Kräftequantum verfügt. Die gefährlichste Zeit aber in dem Krankheitsverlaufe liegt in dem Stadium, in dem die graue Hepatisation ihr Ende erreicht. Es ist dies der Moment, in dem die massenhaft angehäuften abgestorbenen Leukozyten etc. zerfallen und zur Resorption gelangen sollten. Dazu ist aber nach unserer Ansicht vor allem Sauerstoff, d. h. eine starke intensive Zirkulation im kleinen Kreislaufe notwendig. Nun ist aber durch die bereits überstandene Krankheitsperiode das Gefässsystem ausserordentlich in Anspruch genommen und von der Frage, ob seine Leistungsfähigkeit noch eine weitere Steigerung zulasse oder nicht, hängt das weitere Schicksal ab. Ist die Reservekraft noch genügend, so wird stets Lösung rasch erfolgen und in einem Circulus benignus durch die Zunahme der Sauerstoff aufnehmenden Respirationsfläche noch beschleunigt werden. Ist der Vorrat dagegen zu knapp, so wird die Lösung verlangsamt oder bleibt aus, die Respirationsfläche bleibt dieselbe und durch die vergeblichen Anstrengungen des Herzens tritt noch grössere Erschöpfung des Myokards ein. Indem nun aber die toten Zellen weiter bestehen ohne oxydative Zersetzung, treten die anderen autolytischen Prozesse, die auch bei der Verkäsung in Funktion sind, in den Vordergrund und führen zu einer Eindichtung des Exsudates, die nun in einem Circulus vitiosus zur weiteren Erschwerung der Resolution führt. Ausserdem ist nicht zu vergessen, dass möglicherweise auch die Vernichtung der Bazillen und Toxine hierunter verzögert werden kann, ja durch die Ansammlung der toten Zellen vielleicht auch Giftstoffe 
entstehen. Dass hierbei trotz des mangelnden Sauerstoffs sozusagen niemals eine Verkäsung entsteht, mag darauf beruhen, dass eben der Gefässwucherung beim Überstehen der Krankheit in die infiltrierten Bezirke hinein nichts im Wege steht, so dass eine grössere Organisation derselben stattfinden kann. Es entsteht das typische Bild der Karnefikation. Demnach ergibt sich, entsprechend der klinischen Erfahrung, dass vor allem Personen mit herabgesetzter Herzkraft sehr gefährdet sind, wie z. B. Alkoholiker, Fettsüchtige, Nephritiker.

Da aber die Sauerstoffzufuhr nicht nur eine Folge der Zirkulation ist, sondern zweifellos auch abhängt von dem Grade der Sauerstoffladung des zirkulierenden Blutes, so muss, da letztere wiederum proportional ist der Respirationsfläche, auch ein bestimmtes mindest zulässiges Verhältnis bestehen zwischen funktionierendem und infiltriertem Lungenparenchym. Wenn der Prozess deshalb ausgedehnt ist, wird die Prognose eo ipso abgesehen von der Intoxikation und der erschwerten Respiration ungünstiger werden, weil die gefübrliche Resolutionszeit dadurch wesentlich in die Länge gezogen wird. Dass die Pneumonia catarrhalis trotz der kleinen kranken Bezirke so maligne ist, wird neben anderem wohl darauf beruhen, dass sie bei Personen auftritt, die so wie so über geringere zirkulatorische Kräfte verfügen; zweifellos aber gilt auch für sie die lösende Rolle des Sauerstoffes.

Die Pneumonia tuberculosa ist eine der vielen Erscheinungen, die der Tuberkelbazillus zu erzeugen imstande ist. Was für sie gilt, darf daher mehr oder weniger auf alle tuberkulösen Veränderungen bezogen werden und umgekehrt können diese zum Verständnisse jener beitragen. Infolge Vernichtung der Zirkulationsorgane nehmen die unter dem Einfluss des Tuberkelbazillus entstandenen Infiltrate käsige Beschaffenheit an, entsteht die ausgedehnte käsige Pneumonie, die Bronchitis und Peribronchitis caseosa etc., die käsigen Herde der Nebennieren, des Gehirns etc. Selten verschwinden diese Herde oder gehen in Zerfall über, niemals sozusagen in Organen wie die Nebennieren, die in keiner Weise zur Aussenwelt in Beziehung stehen, am häufigsten wohl in den Lungen, wo das resistente Bronchialsystem dem Einflusse der Tuberkelbazillen in geringerem Masse unterworfen ist. Deshalb ist hier Sauerstoffzufuhr ermöglicht auch ohne Gefässbeteiligung. Das an das Bronchiallumen grenzende käsige Gewebe kommt mit Sauerstoff in Berührung und zerfällt infolgedessen in Detritus, der dann ausgehustet wird. Neben der Zufuhr von Sauerstoff kann aber auch Eindringen von Fänlnisbakterien zur Auflösung führen; es ist dies offenbar ein Faktor, der speziell auch bei pneumotuberkulösen Prozessen neben dem erstgenannten berücksichtigt werden muss. Schliesslich kann ja auch, wie oben erwähnt, der Körper 
selbst Mazeration hervorbringen, wenn die Virulenz der Toxine dem Wuchern der Gefässe nicht mehr Einhalt bieten kann und, wie Orth annimmt, das Eindringen von Leukozyten gestattet.

Auf demselben Prinzip wie bei der Tuberkulose beruht nun auch die Verkäsung bei anderen krankhaften Prozessen. Auch die Lues führt bekanntlich zu einer Vernichtung der Gefässe. Schliesslich müssen auch noch die Geschwülste hier Erwähnung finden, da es sich bei ihnen auch oft um schlecht ernährte oder absterbende zerfallende Gewebe handelt. Da die Geschwülste in der Regel mit Gefässen in geringem Grade rersorgt sind oder mit arteriosklerotisch veränderten, wird es sich fragen, ob den destruktiven oxydativen Prozessen kompensierende intrazelluläre aufbauende gegeniiberstehen, was der Fall ist bei kräftigen, vitalen Zellen.

Bei grösseren Tumoren aber finden sich solche Zellen nur in beschränkter Zahl, weil eben die vitale Energie der Geschwulstzellen durch die ungewöhnliche Zellteilung eo ipso herabgesetzt ist, so dass der Quotient sich zugunsten der Mazeration ändert, selbst wenn das Gewebe nur auf die zirkulatorische Sauerstoffversorgung, die bier nicht, wie bei Tuberkulose automatisch unterbunden ist, angewiesen ist. Da aber die geschwächtesten Gewebspartien die ältesten, d. h. die im Zentrum gelegenen sein müssen, so muss auch, wie die Beobachtung lehrt, dort die Erweichung beginnen. Falls aber neben dem Zirkulationssauerstoff auch noch Luft zutreten kann, wird die Zersetzung abgesehen von entsprechenden Mikroorganismen noch viel bedeutender sein müssen.

Wenn man nun das gesamte bisher Dargelegte kurz zusammenfasst, so ergibt sich der Satz:

Die Gesetze der Lösung und Resorption ron abgestorbenen Geweben werden diktiert von der Spannung des anwesenden Sauerstoffes. Da dieser in der Regel durch die Gefässe zugeführt wird, so ist der Einfluss des die Krankheit erzeugenden Momentes auf die Gefässe bestimmend für das Schicksal der betroffenen Gewebe.

Nicht zum mindesten beruht auch hierauf, abgesehen von der bedingten Ernährungsstörung, die Pathogenität der Krankheitserreger, indem durch die ausbleibende Lösung eo ipso jede Tendenz zur Verheilung ausgeschlossen wird.

Die Bedeutung der Gefässe dokumentiertsich daher als primärer Natur, und zwar nach zwei Richtungen hin, der der Ernährung und des Abbaues, oder noch elementarer ausgedrückt: Die Gefässe bestimmen einerseits die assimilierenden, andererseits diedissimilierenden 
Zellprozesse. Diese sind voneinander unabhängig in gewissem Sinne, je nach dem Überwiegen des einen oder anderen tritt Auflösung oder Verquellung ein, sobald die für das Leben zulässige Unterschiedsgrenze überschritten ist.

Beobachtet man nun unter Berücksichtigung dieser primären Rolle der Gefässe den Verlauf der krankhaften Vorgänge, so wird ohne weiteres einleuchten, dass 1 . in vielen Fällen besonders das Verhalten des Zirkulationssystems einen der wesentlichsten Fingerzeige geben muss bei der Prognosestellung, 2. aber auch die Therapie sich vor allem auf die genaue Kontrolle und eventuelle entsprechende Beeinflussung der Gefässe, sei es auf medikamentösem, sei es auf physikalischem Wege stïtzen muss.

Ganz besonders scheint mir dies bei der Pneumonia fibrinosa der Fall zu sein, und zwar speziell dann, wenn die eigentliche Intoxikation das Feld nicht oder nicht mehr beherrscht. Denn gegen die Toxine wird ja voraussichtlich nur ein serotherapeutisches Vorgehen auf Erfolg hoffen dürfen; aus diesem Grunde muss auch die Pneumonia tuberculosa caseosa hier ausser Diskussion fallen, weil bei dieser zuerst die Toxine in Frage kommen. Diese aber tragen in die Physiologie der Zelle einen dritten noch unbekannten Faktor hinein, welcher das gesamte Verhalten wesentlich zu modifizieren imstande ist.

Lässt man aber diesen ausser Frage, wie es bei der Pneumonia fibrinosa in vielen Fällen sicherlich $\mathrm{zu}$ tun gestattet ist, so steht in erster Linie das Verhalten der Gefässe, für deren vitale Energie der Blutdruck wohl in der Regel ein zuverlässiger Indikator ist, sofern andere Momente, die hierbei von Bedeutung sein könnten ${ }^{1}$ ), fehlen. Während nun im allgemeinen beim Pneumoniker dem Herzen hauptsächlich dann Stimulantien zugeführt werden, wenn dasselbe Zeichen schwerer Erschöpfung und drohenden Kollapses zeigt, möchte ich eben unter Berücksichtigung der oben angeführten Theorie der Resolution einer Therapie das Wort reden, die auch bei Fehlen ron Herzstörungen die Zirkulation möglichst anregt, vor allem durch Darreichung von Digitalis. Es ist dies ein Gedanke, den schon Bierner ausgesprochen und empirisch gefunden hat, gestützt auf seine Beobachtungen am Krankenbette.

Die Biermersche Theorie hat aber nicht nachhaltigen Beifall gefunden; erst in neuerer Zeit wurde sie durch Petrescu ${ }^{2}$ ) [p. 214] wiederum empfohlen und hat namentlich durch Maragli a no $\left.{ }^{2}\right)$ [p.214]

1) Sahli, Über das absolute Sphygmogramm und seine klinische Bedeutung, nebst kritischen Bemerkungen über einige neuere sphygmomanometrische Arbeiten. Leipzig. Deutsch. Arch. f. klin. Med. LXXXI. Bd. 1904. p. 498.

2) P. K. Pel, Die Behandlung der krupösen Pneumonie. Samml. klic. Vorträge v. Volkmann. Nr. 284. Leipzig. 1900. 
warme Würdigung gefunden. Während aber dieser Forscher geradezu eine spezifische Wirkung, gewissermassen ein Antitoxin, im Digitalis finden will und deshalb die Verabreichung in ausserordentlichen Dosen empfiehlt, möchte ich den durch die klinischen Erfahrungen dieser Forscher sichergestellten günstigen Digitaliseinfluss nicht sowohl einer spezifischen Wirkung zuschreiben, als vielmehr dem durch Digitalis angefachten und angeregten Gefäss- und Herztonus, dessen Konsequenz unter Beiziehung des Dargelegten sich ohne weiteres ergibt. Da aber eine Sauerstoffversorgung der Lungen vor allem dann besonders vonnöten ist, wenn der Entzündungsprozess mehr weniger zum Stillstand gekommen ist und die Lösung des Exsudates vor sich gehen sollte, so ist eine Anregung der Zirkulation vor allem erforderlich gegen Ende des Stadiums der grauen Hepatisation. Während man also vorher mehr abwartend sich verhält und nur bei drohenden Symptomen von seiten des Kreislaufs eingreift, wird man jetzt Digitalis verabreichen, um sowohl das Schlagvolumen als den Gefässtonus in dieser kritischen Zeit zu steigern. Durch Digitalis wird bekanntlich nach $\mathrm{Cl}_{\text {oetta-Filehn }}{ }^{1}$ ) die Reservekraft des Herzens in Anspruch genommen und eingesetzt. Zweifellos ist es nun erlaubt, für eine kurze aber kritische Zeit ein Organ besonders anzustrengen, wenn dadurch die Rekonvaleszenz beschleunigt wird, in der die Gelegenheit zum Ersatz und zur Erholung sich reichlich findet. Die Therapie würde damit nur das Beispiel nachahmen, das der Organismus oft selber gibt. Schliesslich liesse sich noch erwägen, ob es nicht, namentlich in Fällen mit geringer Respirationsfläche, gelegentlich angezeigt wäre, durch Erzeugung hyperämischer Hautflächen im Stadium der grauen Hepatisation die O-Ladung des Blutes zu heben.

Etwas schwieriger liegen, wie erwähnt, die Verhältnisse für ein therapeutisches Eingreifen bei der Pneumonia tuberculosa, indem hier die Toxine im Vordergrund stehen, die übrigen Momente aber erst in zweiter Linie kommen. Dagegen ist immerhin auch hier zu bedenken, dass die Intensität des Toxins ein relativer Begriff ist, bezogen auf den Massstab "Reaktionsfähigkeit“ des Organismus. Wird dieser gehoben, so nimmt der erste Wert ab. Nun ist aber bei der Tuberkulose vor allem darin ein Circulus vitiosus zu sehen, dass der Tuberkelbazillus im besonderen die Gefässe schädigt. Dadurch wird seine Wirkung nicht nur ungehemmter, sondern durch die Bildung eines nekrotisch-käsigen Walles wird die Vernichtung des Erregers bedeutend erschwert. Das therapeutische Interesse

1) Cloetta-Filehne, Lehrbuch der Arzneimittellehre. X. Aufl. Tübingen. 1901. p. $213 \mathrm{ff}$. 
muss sich daher speziell darauf richten, einmal, wie die Empirie schon längst gezeigt, den Organismus zu kräftigen, dann aber, ganz besonders, die Bildung und Wucherung der Gefässe anzuregen, d. h. also, spezielle Organe im Organismus zu kräftigen. Welche Mittel hier in Betracht kommen dürften, bleibt der weiteren Forschung vorbehalten; es darf aber schon jetzt angeführt werden, dass der relativ spezifische Einfluss der Jodpräparate - wie z. B. Jodoformöl und neuere - vielleicht mit Recht auf die besonders die Gefässe beeinflussende Wirkung des Jods zurückgeführt werden.

Ein weiterer Weg wäre ferner, den Infiltrationsherd dadurch anzugreifen, dass man das Blut, das bis an dessen Grenze gelangt, besonders aktiviert, so dass eine lebhaftere Verbrennung und Zersetzung stattfinden muss. Auf diesem Prinzip beruhen offenbar zum Teil die Erfolge der Staungstherapie nach Bier bei tuberkulösen Gelenkaffektionen z. B.

$\mathrm{Ob}$ die Biersche Methode sich auch auf innere Organe anwenden lassen wird, bleibt freilich noch der Zukunft zur Entscheidung ïberlassen. Doch scheinen nach den Angaben von $\mathrm{Bie} \mathrm{r}^{1}$ ) die bisherigen Versuche in dieser Richtung nicht ohne Erfolg zu sein. Zweifellos aber wäre bei der Pneumonia tuberculosa vor allem eine solche Behandlung angezeigt und dürfte nach obigem auch bei der Pneumonia crouposa Erfolg versprechen, offenbar mehr als die bisherige, roh empirisch tastende Wickeltherapie. Es wäre demnach $\mathrm{zu}$ hoffen, dass auch für die mehr abwartende Therapie der Pneumonia crouposa und tuberculosa eine Zeit aktiveren Eingreifens anbrechen könnte.

Zum Schlusse möchte ich mir noch zu bemerken erlauben, dass diese Darlegung nicht beansprucht die Fragen endgültig zu beantworten. Es ist dies vielmehr ein vielleicht etwas einseitiger Schluss von allgemein biologischem Gesichtspunkte aus auf speziell pathologische Verhältnisse. Doch schien er mir etwelche Berechtigung zu haben, weil damit eben auf Grund des allgemeinen Prinzips mühelos eine umfassende Erklärung sich finden lässt, die weitere Forschungen über die Wirksamkeit des Tuberkelbazillus sowohl als über die zytologische Zusammensetzung der in Frage kommenden Exsudate in keiner Beziehung ausschliesst, sondern dadurch nur eine weitere Ergänzung und Vertiefung erfahren kann.

Zum Schlusse sei es mir gestattet, Herrn Prof. Dr. Ernst für die Förderung und das Interesse an dieser Arbeit wärmstens zu danken.

1) Bier, Hyperämie als Heilmittel. 